\title{
A Case of Turner Syndrome with Multiple Embolic Infarcts
}

\author{
Cindy W. Yoon Eungseok Lee Byung-Nam Yoon Hee-Kwon Park \\ Joung-Ho Rha \\ Department of Neurology, Inha University School of Medicine, Incheon, South Korea
}

\section{Keywords}

Turner syndrome $\cdot$ Stroke $\cdot$ Embolic infarct $\cdot$ Cryptogenic embolism

\begin{abstract}
Only a few cases of Turner syndrome (TS) with ischemic stroke have been reported. Various arteriopathies of the cerebral arteries, including fibromuscular dysplasia, congenital hypoplasia, moyamoya syndrome, and premature atherosclerosis have been assumed to be the cause of ischemic stroke in TS. There has been no case report of a TS patient presenting with an embolic stroke pattern without any cerebral arteriopathy. A 28-year-old woman with TS was referred to our hospital because of abnormal brain magnetic resonance imaging (MRI) findings. She underwent brain MRI at the referring hospital because she experienced suddenonset diffuse headache. Diffusion-weighted imaging revealed multiple acute embolic infarcts in different vascular territories. Intracranial and extracranial arterial disease was not detected on cerebral magnetic resonance angiography and carotid sonography. Embolic source workups, including transthoracic and transesophageal echocardiography, Holter monitoring, and transcranial Doppler shunt study, were all negative. Hypercoagulability and vasculitis panels were also negative. Our patient was diagnosed with cryptogenic embolic stroke. This is the first report of a TS patient with an embolic stroke pattern. Our case shows that ischemic stroke in TS could be due to embolism as well as the various cerebral arteriopathies documented in previous reports.




\section{Introduction}

Turner syndrome (TS) is caused by partial or complete monosomy of the $\mathrm{X}$ chromosome [1]. Only a few cases of TS with ischemic stroke have been reported [2-5]. In previously reported cases, various arteriopathies affecting the cerebral arteries such as fibromuscular dysplasia [2], hypoplasia [3], moyamoya syndrome [4], and premature atherosclerosis [5] were assumed to be the causes of ischemic stroke in TS. Ischemic stroke with an embolic pattern has not yet been reported in TS. Here, we report a case of TS with multiple embolic infarcts caused by a cryptogenic embolism.

\section{Case Presentation}

A 28-year-old woman with TS was referred to our hospital because of abnormal findings on brain magnetic resonance imaging (MRI). She was diagnosed with TS [karyotype: 45, $\mathrm{X} / 46, \mathrm{X},+\mathrm{mar}]$ at the age of 16 years due to amenorrhea. She denied alcohol, tobacco, or drug use and had no family history of neurologic disorders. The patient was afebrile with a blood pressure of $180 / 130 \mathrm{~mm} \mathrm{Hg}$ and a heart rate of 89 beats $/ \mathrm{min}$. She was obese with a body mass index of $27.3 \mathrm{~kg} / \mathrm{m}^{2}$ (160 cm height, $70 \mathrm{~kg}$ weight). Neurological examination revealed no focal deficits.

She visited hospital due to sudden-onset severe headache with high blood pressure (240/140 mm Hg). She underwent brain computed tomography and MRI at the referring hospital for differential diagnosis of thunderclap headache including hypertensive intracranial hemorrhage, subarachnoid hemorrhage, arterial dissection, or reversible cerebral vasoconstriction syndrome. Brain computed tomography was unremarkable, but diffusionweighted imaging showed restricted diffusion in multiple lesions involving multiple vascular territories (fig. 1a). Cerebral magnetic resonance angiography (MRA) (fig. 1b) and carotid sonography demonstrated no extracranial or intracranial arterial stenosis. Chest X-ray and electrocardiography were unremarkable. Laboratory tests revealed diabetes mellitus (fasting plasma glucose $219 \mathrm{mg} / \mathrm{dl}$, hemoglobin $\mathrm{A}_{1 \mathrm{C}}$ 11.0\%) and hyperlipidemia (low-density lipoprotein $189 \mathrm{mg} / \mathrm{dl}$ ). Transthoracic echocardiography revealed normal valvular structure and function with a normal left ventricular ejection fraction. Patent foramen ovale, atrial septal aneurysm, or any aortic pathologies including aortic atheroma were not detected in transesophageal echocardiography. Holter monitoring and transcranial Doppler shunt study also failed to find the embolic source. The results of the hypercoagulability panel, vasculitis panel, and cerebrospinal fluid examination were all negative. Our patient was diagnosed with cryptogenic embolic stroke.

Aspirin was administered, and medications for hypertension, hyperlipidemia, and diabetes were also started. Within few hours after blood pressure control, headache was resolved without any pain medication. The patient was discharged from the hospital without any symptoms of stroke, and recurrence was not observed in the 2 years after discharge.

\section{Discussion}

There have been no cohort studies on the etiology of ischemic stroke in TS. Only a few case reports have described individual causes of ischemic stroke. Cerebrovascular arteriopathies such as fibromuscular dysplasia [2], congenital hypoplasia [3], moyamoya syndrome 


\section{Case Reports in Neurology}

Yoon et al.: A Case of Turner Syndrome with Multiple Embolic Infarcts

[4], and premature atherosclerosis [5] have been shown to be causes of ischemic stroke in previous case reports. In this case, there was no evidence of cerebrovascular arteriopathy on brain MRA or carotid sonography. Furthermore, our patient showed multiple lesions in different vascular territories that were strongly suggestive of embolic stroke.

Atrial fibrillation (AF) could also be considered a potential source of embolic stroke in TS, as P-wave dispersion, a potential substrate for AF [6], is increased in TS [7]. The classical risk factors for developing AF include hypertension, diabetes mellitus, and valvular disease, and these are more common in TS than in the general population. We did not detect AF on 24-hour Holter monitoring in our patient. However, we could not completely exclude the possibility of paroxysmal AF, as Holter monitoring has a low yield for AF detection (3-5\%) [8]. Other possible cardio-aortic sources of embolism include intracardiac shunts, thrombi [9], calcifications/vegetations in the mitral valve, or aortic atheroma plaque [10]. We sought to identify possible cardio-aortic sources on transthoracic echocardiography, transesophageal echocardiography, and transcranial Doppler shunt test; however, all tests were negative.

A hypercoagulable state could be considered a contributing factor of thromboembolism in TS. Some case reports have described deep venous thrombosis and portal vein thrombosis in TS [11, 12]. Abnormalities in clotting and fibrinolytic factor levels are seen in some TS patients. These factors include fibrinogen, D-dimer, factor VIII, von Willebrand factor, and proteins C and S [13]. We also considered the possibility of hypercoagulability as a cause of stroke in this case; however, levels of D-dimer, fibrinogen, protein C and S were normal. She did not complain of any symptoms suspicious for systemic thromboembolism.

The embolic source of stroke in our patient remains unclear; however, this is the first report of a TS patient with an embolic stroke pattern. Our case suggests another possible mechanism of stroke in TS that is different from previous case reports. Ischemic stroke in TS could be due to embolism as well as various cerebral arteriopathies. Further investigation is needed to more fully understand the various mechanisms of ischemic stroke in TS.

\section{Conclusion}

Ischemic stroke in TS could be due to embolism as well as various cerebral arteriopathies, as documented in previous reports. Further studies are needed to determine the extent of the underlying mechanisms of ischemic stroke in TS.

\section{Statement of Ethics}

Informed consent was obtained from the patient. This case report has been approved by the Institutional Review Board of the Inha University Hospital.

\section{Disclosure Statement}

The authors have no conflicts of interest to disclose. 


\section{References}

1 Lemli L, Smith DW: The XO syndrome: a study of the differentiated phenotype in 25 patients. J Pediatrics 1963;63:577-588.

2 Lancman M, Mesropian H, Serra P, Granillo R: Turner's syndrome, fibromuscular dysplasia, and stroke. Stroke 1991;22:269-271.

-3 Komori H, Matsuishi T, Abe T, Nagata Y, Ohtaki E, Kojima K, et al: Turner syndrome and occlusion of the internal carotid artery. J Child Neurol 1993;8:412-415.

4 Spengos K, Kosmaidou-Aravidou Z, Tsivgoulis G, Vassilopoulou S, Grigori-Kostaraki P, Zis V: Moyamoya syndrome in a Caucasian woman with Turner's syndrome. Eur J Neurol 2006;13:e7-e8.

-5 Irioka T, Mizusawa H: Ischemic stroke in a young adult with Turner syndrome. Neurol Sci 2011;32:317-319.

-6 Dilaveris PE, Gialafos JE: P-wave dispersion: a novel predictor of paroxysmal atrial fibrillation. Ann Noninvasive Electrocardiol 2001;6:159-165.

7 Sozen AB, Cefle K, Kudat H, Ozturk S, Oflaz H, Pamukcu B, et al: Atrial and ventricular arryhthmogenic potential in Turner Syndrome. Pacing Clin Electrophysiol 2008;31:1140-1145.

-8 Liao J, Khalid Z, Scallan C, Morillo C, O'Donnell M: Noninvasive cardiac monitoring for detecting paroxysmal atrial fibrillation or flutter after acute ischemic stroke: a systematic review. Stroke 2007;38:2935-2940.

-9 Bakiler AR, Cangar S, Aksit S, Unver A, Yaprak I, Caglayan S: Disappearing intracardiac thrombi in both atria after mumps in a patient with Turner syndrome. Chest 1993;103:1611-1612.

10 Sybert VP: Cardiovascular malformations and complications in Turner syndrome. Pediatrics 1998;101:e11.

11 Jobe S, Donohoue P, Di Paola J: Deep venous thrombosis and Turner syndrome. J Pediatr Hematol Oncol 2004;26:272.

12 Kopacek Zilz C, Keller Brenner J, Elnecave RH: Portal vein thrombosis and high factor VIII in Turner syndrome. Horm Res Paediatr 2006;66:89-93.

-13 Calcaterra V, Gamba G, Montani N, De Silvestri A, Terulla V, Lanati G, et al: Thrombophilic screening in Turner syndrome. J Endocrinol Invest 2011;34:676-679.

C.W.Y. and E.L. contributed equally to this work 


\section{Case Reports in Neurology}

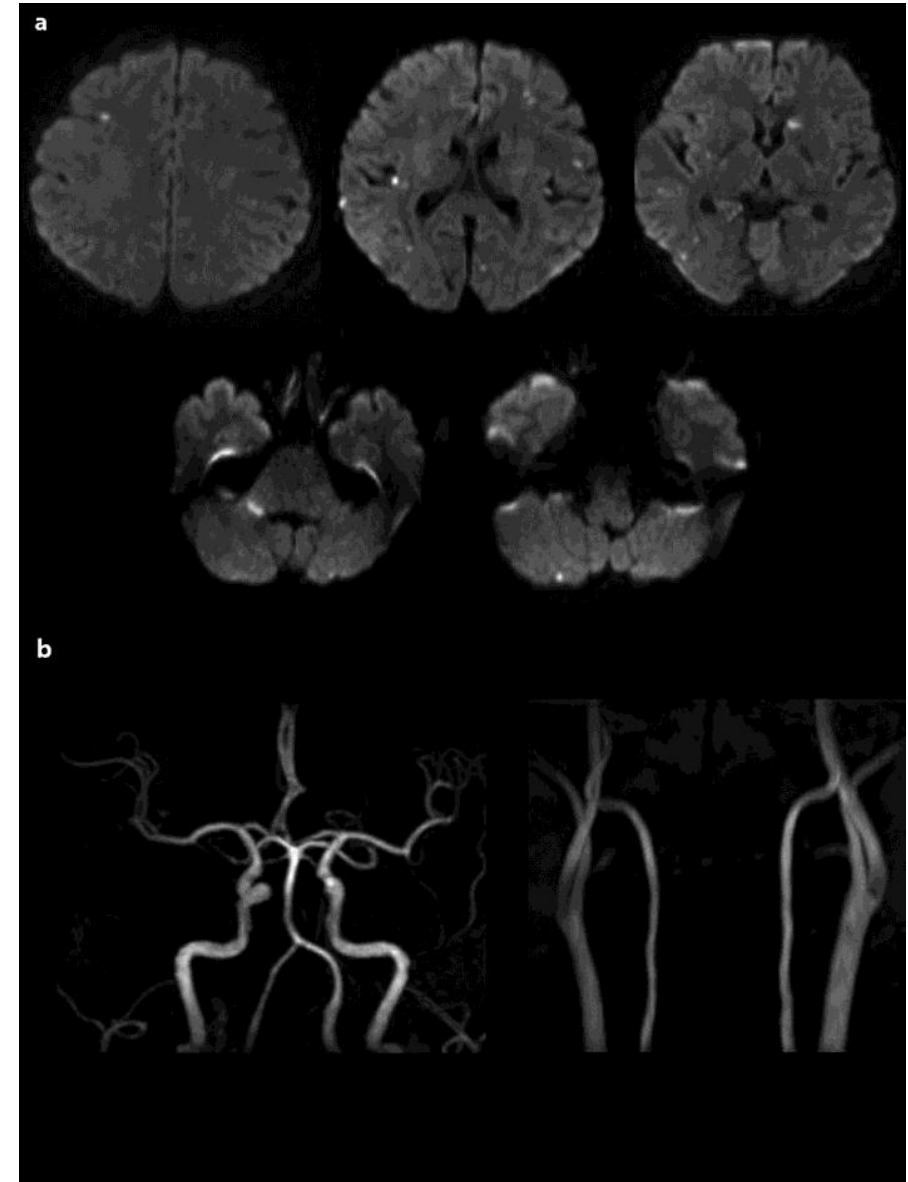

Fig. 1. MRI findings. a Diffusion-weighted imaging shows scattered lesions involving multiple vascular territories. $\mathbf{b}$ No stenosis is visible on MRA of the intracranial and extracranial arteries. 